ture. From numerous experiments it follows that the gamma fibres, which are responsible for the basic reflex arch that controls the muscle tone, are much thinner than the motor and sensory fibres.

It is common knowledge too that the nerve fibres, depending upon their thickness, have different susceptibility to damaging factors. Therefore, if the root or peripheral nerve has to be treated with an appropriate phenol solution it would result in damaging selectively the thin gamma fibres and 'the spastic arch' would be interrupted at that level.

Our own clinical material consists of 15 patients in whom unilateral or bilateral phenolisation of spinal roots was performed and of 23 patients in whom 49 peripheral nerves were phenolised. From our experience it can be concluded that in cases of complete paraplegia of the lower extremities accompanied by mass reflex synergy preventing automatic urination, subarachnoid injection of phenol solution is the method of choice. Phenol blocks of the peripheral nerves, however, are recommended in patients with spastic paresis of lower extremities without sphincter disturbance and in particular in patients with hemiparesis associated with flexion contractures of the upper extremity.

\title{
PERIPHERAL NERVE AND MOTOR POINT PHENOL BLOCKS FOR SPASTICITY
}

\author{
By H. D. Cain, A. Glass, J. Spiegler, H. Liebgold and S. Mead \\ Kaiser Foundation Rehabilitation Centre, Vallejo, California, U.S.A.
}

REDUCtion of spasticity in the extremities due to Central Nervous System diseases or disorders has been achieved by application of small amounts of 3 to 6 per cent. aqueous phenol solution to the peripheral nerve or motor points of the involved muscles. Seventy patients, ranging in age from 6 to 73, have been treated in the past I 8 months. This simple technique is performed using an electrical stimulator which delivers a pulsating direct current through a hypodermic needle that is coated with teflon except for its tip. The peripheral nerve or motor point can first be located externally using a surface monopolar electrode. The teflon-coated needle electrode is then inserted through the skin site and visible muscle contractions occurring with minimal current intensity designates the respective peripheral nerve or motor point. From 0.3 to $0.5 \mathrm{ml}$. of aqueous phenol is injected at the motor point level or one to $10 \mathrm{ml}$. at the peripheral nerve level. Repetition of injections may be necessary for desired results. Spasticity may partially return in weeks, but satisfactory reduction for over one year has been observed. No objective or subjective sensory or motor loss has yet occurred. Mild increase in voluntary motor function has been noted in certain instances after spasticity reduction. Electromyographic studies have not revealed evidence of complete denervation after the phenol blocks. The technique is simple and effective in reducing spasticity. Film demonstration. 


\title{
Discussion
}

Apolinario, E. (Argentine). Our experience with the procedure of motor point block is not very large so far. We used it only in a few cases with incomplete lesions where muscle power and sensation must be preserved. The procedure we use is more or less the same as described by Dr. Glass, but we use weaker concentrations and we will keep his higher concentrations in mind for the future because our results are not very long lasting. We usually perform an electromyography of the muscles to be blocked before any procedure was applied. Then a test with procaine solution is made at a concentration of 0.5 to I per cent. and thenthe electromyographic record is repeated. If the results are satisfactory we proceed with the definite blocking with 2 per cent. aqueous phenol solution only but in future, we will use stronger concentrations.

\section{PATHOLOGICAL FINDINGS FOLLOWING THE INTRATHECAL INJECTION OF ETHYL ALCOHOL IN MAN}

\author{
By J. Trevor Hughes, M.D. \\ From the Oxford Hospitals and the National Spinal Injuries Centre, Stoke Mandeville \\ Hospital, Aylesbury
}

THE therapeutic injection of alcohol into the subarachnoid space in man for the treatment of pain was first described by Dogliotti (I93I), and the similar use of phenol dates from the report of Maher (1955). These therapeutic injections were devised for the relief of chronic pain, but subsequently both substances have been used to relieve the painful spasms associated with paraplegia. Whilst the chemical toxic effect of the two agents is very similar, the method of administration differs. Alcohol being hypobaric with respect to cerebrospinal fluid rises in the subarachnoid space and, to affect the caudal part of the spinal cord and its roots, the alcohol is given slowly (to avoid rapid mixing with C.S.F.) with the patient in the prone head-down position (Tank, et al., 1963). Phenol is given mixed with a radio -opaque solution such as myodil and the resultant heavy mixture descends in the spinal cord and affects the dependent part of the nervous system.

In the treatment of chronic intractable pain phenol is often preferred to alcohol and it is probable that with the careful positioning of the patient a more selective effect can be obtained with phenol. However, in the special problem of intractable spasticity in paraplegia and tetraplegia it has been found by Guttmann (1946, I953), Sheldon and Bors (1948) and others that alcohol gives most satisfactory clinical results. It is not my intention to discuss the clinical use of these agents, but to give my own observations on the resulting pathological changes. There have been several detailed reports on the pathological findings after the subarachnoid injection of phenol (Berry \& Olszewski, I963; Smith, I964) but fewer and less detailed observations on the findings in alcohol injection. Consequently my own findings may be of value since they are based on cases from Stoke Mandeville Hospital where intrathecal alcohol has been extensively used since I 946.

The principal clinical features of the six cases which have been studied in 